\title{
Expérimentation d'un EVE pour la co-création spatiale et pédagogique
}

\section{Experimenting an EVE for spatial and educational co- creation}

\author{
Sandro Varano ${ }^{1 *}$, Nicolas Descamps ${ }^{1}$ et Eric Touvenot ${ }^{2 * *}$ \\ ${ }^{1}$ AMUP (EA 7309), ENSA Strasbourg, 6-8 Boulevard Wilson 67000 Strasbourg, France \\ ${ }^{2}$ CRAI (UMR MAP 3495), ENSA Nancy, 2 rue Bastien Lepage 54000 Nancy, France
}

\begin{abstract}
Résumé. Dans la continuité du projet de recherche Dune-EOLE portant sur la transformation numérique de l'enseignement supérieur, l'ENSA Strasbourg expérimente l'utilisation d'un Environnement Virtuel Éducatif (EVE) pour la co-création spatiale et pédagogique. L'objectif est de réinterroger les modalités et espaces d'apprentissage, les pratiques pédagogiques à l'aide de dispositifs immersifs. Nous supposons que l'utilisation de la réalité virtuelle constitue une entrée à cette problématique et pourrait favoriser une forme d'autodidaxie, de collaboration entre pairs et un séquençage entre espaces physique et virtuel. L'article explicite notre approche expérimentale, menée avec des étudiants et des enseignantschercheurs, ainsi que les réflexions portant sur le rôle de l'enseignant, les modalités d'apprentissage et les systèmes de représentation.
\end{abstract}

Mots-clés. EVE, espace d'apprentissage, dispositif pédagogique, réalité virtuelle.

\begin{abstract}
In continuation of the Dune-EOLE research project on the digital transformation of university education, the ENSA Strasbourg are experimenting the use of an Educational Virtual Environment (EVE) for spatial and educational co-creation. The objective is to re-examine learning methods and spaces, teaching devices using immersive tools. We assumed that the use of virtual reality constitutes an entry to this problem and could favor a form of self-learning, collaboration between peers and a sequencing between physical and virtual spaces. The article explains our experimental approach, carried out in collaboration with students and teacherresearchers, as well as the reflections that emerged, about the role of the teacher, learning methods and representation systems.
\end{abstract}

Keywords. EVE, learning space, educational device, virtual reality.

\footnotetext{
*sandro.varano@strasbourg.archi.fr ; nicolas.descamps@strasbourg.archi.fr

** eric.touvenot@nancy.archi.fr
} 


\section{Introduction}

Cet article s'inscrit dans la continuité des travaux menés dans le cadre du projet de recherche Dune-EOLE ${ }^{1}$, financé par l'Agence Nationale de la Recherche ${ }^{2}$ et co-coordonné par les Universités de Strasbourg et de Lorraine ${ }^{3}$ depuis 2016. L'objectif de la mission EOLE est d'interroger les nouvelles modalités d'apprentissage et d'enseignement avec le numérique et d'accompagner «la transformation numérique des établissements d'enseignement supérieur dans le cadre du programme Investissements d'Avenir ${ }^{4}$.

Cette mission de recherche a été scindée en 3 axes concernant 1) Les espaces d'apprentissage ; 2) Les moteurs de la transformation et 3) Le compte numérique de formation. Dans le cadre de l'axe 1 visant à «consolider et élargir les espaces d'apprentissage, des campus connectés aux campus virtuels $»^{5}$, les Ecoles Nationales Supérieures d'Architecture (ENSA) de Strasbourg et Nancy ont été mises à contribution. La participation des ENSA dans la réflexion sur les espaces d'enseignement en lien avec le numérique s'explique entre autre par le fait que les espaces architecturaux, « en tant que cadres physiques de l'apprentissage, sont [...] indissociables des processus humains de construction des savoirs et des apprentissages » (Mazalto \& Paltrinieri, 2013) et conditionnent les pratiques pédagogiques.

A l'aune des premiers résultats du projet Dune-EOLE et des recherches universitaires existantes sur les espaces d'apprentissages mettant en évidence les relations entre espaces architecturaux et pédagogies, l'objectif est de questionner «l'introduction d'artefacts numériques dans une fonction de médiation» (Delépine 2018, 242) et de comprendre comment la culture numérique - plus que les technologies numériques - considérée « en tant que productrice d'artefacts, notamment spatiaux, [...] ont une incidence sur les modalités pédagogiques et la nature de l'apprentissage » (Valenzuela-Aguilera 2018, 15).

Ainsi, en considérant les évolutions numériques dans le cadre d'une mutation non seulement technologique mais également culturelle (Ibidem, 13), nous souhaiterions savoir comment l'usage du numérique et plus spécifiquement de la réalité virtuelle, redéfinit les modalités d'apprentissage et d'enseignement dans la co-création d'espaces architecturaux ; la conception d'espaces impliquant une collaboration entre enseignants-chercheurs et étudiants à travers l'utilisation d'outils et instruments médiateurs.

La création d'un Environnement Virtuel Éducatif (EVE) conçu pour la co-création spatiale pourrait nous permettre d'adapter les espaces existants aux nouvelles approches pédagogiques dans une démarche de Design Thinking en considérant l'étudiant comme «designer as a self organizing system » (Jones 1970, 46) : la notion de designer comme auto-organisateur « implique l'usage de la pratique réflexive, lors de laquelle le concepteur va se prendre comme objet de sa propre réflexion afin d'expliciter la façon dont la démarche intellectuelle est organisée permettant de la formaliser et de la rendre compréhensible » (Descamps, 2017).

Dans cet article, nous reprendrons la définition proposée par Loup-Escande, Jamet et al. : «Un EVE peut être défini comme un environnement virtuel - fondé sur un modèle pédagogique spécifique en fonction d'objectifs didactiques - qui fournit aux utilisateurs des

\footnotetext{
${ }^{1}$ « Développement d'universités numériques expérimentales » et « Engagement pour ouvrir l'éducation ».

${ }^{2}$ Projet ANR-16-DUNE-0001-EOLE.

${ }^{3}$ Le projet Dune-EOLE est réalisé en partenariat avec l'UHA, l'URCA, UNIT et les ENSA Strasbourg et Nancy

${ }^{4}$ Voir les axes de recherche et les résultats Dune-EOLE sur : http://www.dune-eole.fr/laureatsdune/\#page-content

${ }^{5} \mathrm{http}: / / \mathrm{www} . d u n e-e o l e . f r / a x e-1-l e s-e s p a c e s-d a p p r e n t i s s a g e / \# p a g e-c o n t e n t$
} 
expériences qu'ils ne peuvent pas trouver (totalement ou partiellement) dans le monde réel et qui favorise des apprentissages chez les apprenants. » (Loup-Escande, Jamet et al. 2015)

Notre EVE est ainsi considéré comme un artefact numérique et interactif qui permet à l'étudiant/apprenant d'être immergé dans un environnement virtuel pour expérimenter des situations diverses. Il est un instrument qui « remplit des fonctions plus complexes, à la fois techniques et sémiotiques » (Albero 2010a, 15), autour duquel s'organisent l'enseignement et l'apprentissage ; il constitue dès lors une séquence d'apprentissage qui s'inscrit dans un dispositif pédagogique plus large, celui qui « englobe les lieux, les méthodes et l'ensemble fonctionnel des acteurs et des moyens mobilisés en vue d'un objectif » (Albero 2010b, 1).

L'approche instrumentale de Pierre Rabardel, nous montre comment les instruments sont façonnés par la technologie et ses utilisateurs (Rabardel, 1995). Il distingue deux processus mêlés : «instrumentation » et «instrumentalisation »; le premier met en avant la façon dont l'instrument conditionne les actions de l'utilisateur pour réaliser sa tâche; le second implique une « contribution » de l'utilisateur pour la «personnalisation », la « différenciation », le « détournement » de l'instrument.

Nous présupposons qu'un EVE pourrait être un instrument original, qui ne viendrait pas se substituer mais compléter les outils de projet et de représentation que sont la maquette, le plan, la coupe, le discours, ... La réalité virtuelle pourrait ainsi permettre une nouvelle perception, au moins visuelle et auditive, du projet architectural à l'échelle $1 / 1$. Cela dans le but de mettre en évidence les notions d'échelle, d'ambiance, de luminosité et d'usage avant la présentation finale, voire la construction.

Dans un premier temps, nous passerons en revue des projets développés dans le cadre de la mission de recherche Dune-EOLE mettant en avant l'influence réciproque des espaces d'apprentissage avec l'enseignement.

Nous nous intéresserons ensuite aux EVE développés au sein des ENSA avec «l'idée que la réalité virtuelle en y intégrant l'importance d'une conception pédagogique devient un outil à part entière dans le cadre de l'enseignement » (Merchant et al., 2014) et participe à certaine forme d'autodidaxie de la part des étudiants.

Nous conclurons en analysant les premiers résultats en matière de pédagogie et de perspectives de conception spatiale. De par son côté expérimental, ce projet d'espaces pédagogiques immersifs continue d'évoluer ce qui nous permettra également d'émettre des pistes de recherches et d'améliorations de la version présentée dans cet article.

\section{Expérimentations de conception de NEA dans le cadre du projet Dune-EOLE}

\subsection{Des premières réflexions sur le rapport spatialité/pédagogie}

Parallèlement à la constitution d'un État de l'art des Nouveaux Espaces d'Apprentissage (NEA) (Descamps, 2018), deux enseignants-chercheurs en ENSA, ont mis en place une réflexion prospective grâce à des cours optionnels de Master afin de questionner et d'expérimenter l'aménagement de NEA et leurs pédagogies associées. En 2018 et 2019, dans le cadre de l'atelier «Modélisation/Fabrication » (M1 - ENSAS) les étudiants ont proposé des dispositifs d'apprentissage numérique, mobile et connecté capables de se «greffer» dans un lieu universitaire existant ${ }^{6}$. En 2019, au sein de l'atelier «Laboratoire Lumière: ambiances et immersions », leurs camarades ont réfléchi à la

\footnotetext{
${ }^{6}$ Rendu final des travaux et soutenances publiques des étudiants les 14 janvier 2019 et 20 janvier 2020 à l'ENSAS ; PDF disponible sur le site Dune-EOLE : http://www.dune-eole.fr/actualites/\#pagecontent
} 
conception d'ambiances d'apprentissage afin de réaménager un hall de l'Université de Strasbourg et une salle de l'IUT de Colmar' ${ }^{7}$ Les projets d'étudiants ont été présentés en réalité virtuelle lors de la réunion annuelle du programme Dune-EOLE aux membres du consortium à l'Université de Reims Champagne Ardenne ${ }^{8}$.

Les enseignements avaient pour objectif de réinterroger les méthodes pédagogiques en considérant une autodidaxie, une volonté de collaboration et de fluidité spatio-temporelle permise par le numérique, nécessitant un changement dans l'approche pédagogique de la part des enseignants (Loup-Escande, Jamet et al. 2015, 1). Ainsi, l'existence de ces enseignements pragmatiques se justifie par notre double posture d'enseignants-chercheurs et d'architectes qui nous fait nous interroger sur « le rôle propre des espaces pédagogiques tant du côté de la forme que de celui de l'usage » (Touvenot \& Varano, 2019) mais également par les approches pédagogiques particulières des études en architecture. En effet, la dimension de Learning by doing, c'est-à-dire un « apprentissage [qui] combine savoir et manipulation objet» (Weber, Rodhain \& Fallery 2019, 41) y est déjà prédominante notamment dans la pratique du projet. Dans cette partie nous présenterons succinctement deux projets issus de ces enseignements.

\subsection{Création d'espaces et de pratiques didactiques en réalité virtuelle}

\subsubsection{Error 404, (projet d'étudiants ${ }^{9}$ 2018-2019 à l'ENSAS)}

Le projet Error 404 est pertinent dans notre étude car il témoigne d'une réflexion sur les séquences d'apprentissage, sur la complémentarité des espaces physiques et virtuels ${ }^{10}$, de la co-création d'une architecture virtuelle impermanente et d'un apprentissage par et pour les étudiants. Le projet a pour objectif de questionner l'importance des cinq sens dans la conception et dans la réception d'une architecture grâce à la création d'un espace virtuel immersif mouvant, augmenté par un espace physique également évolutif. L'intérêt majeur est d'avoir voulu dépasser la domination visuelle très souvent mise en avant dans les systèmes immersifs car considérée comme «le sens dominant de la perception humaine » (Mellet d'Huart 2001, 332) et de renforcer la dimension haptique de l'immersion. Les étudiants ont en effet choisi de retranscrire l'ensemble des sens, en les superposant, dans et par l'espace à l'aide de pistons déformants les parois souples des murs et du plafond. Si la traduction des perceptions sensorielles en modulations spatiales est d'une subjectivité assumée, les étudiants ont mis en avant une réflexion sur la place des sens en architecture ainsi qu'une pratique réflexive dans la manière d'aborder le projet, illustrée par de nombreux diagrammes conceptuels concernant aussi bien l'espace que leur processus de création (Figure 1). Ils ont également interrogé les notions de complémentarité, de singularité et de confrontation entre espaces physiques et virtuels et dans les modes de représentation (réalité virtuelle, maquettes, diagrammes, plans, coupes, croquis et

\footnotetext{
${ }^{7}$ Rendu final des travaux et soutenance publique des étudiants le 3 juin 2019 à l'ENSAS ; PDF disponible sur le site Dune-EOLE : http://www.dune-eole.fr/actualites/\#page-content

${ }^{8}$ Démonstrations du 9 octobre 2019. Voir à ce sujet le poster et le livret de présentation des travaux d'étudiants des ENSAS et ENSAN sur le site Dune-EOLE : http://www.dune-eole.fr/actualites/\#pagecontent

${ }^{9}$ BALLET Mélanie, BIACHE Gaël \& COUTANT Tom

${ }^{10}$ Dans cet article nous parlerons d'espaces physiques et virtuels et non de réels/virtuels considérant comme Gilles Deleuze que «Le virtuel possède une pleine réalité, en tant que virtuelle » (Différence et Répétition, PUF, 2011).
} 
discours $)^{11}$ dans une logique de séquençage de l'apprentissage qui commence dans l'espace physique et se poursuit dans l'espace virtuel.

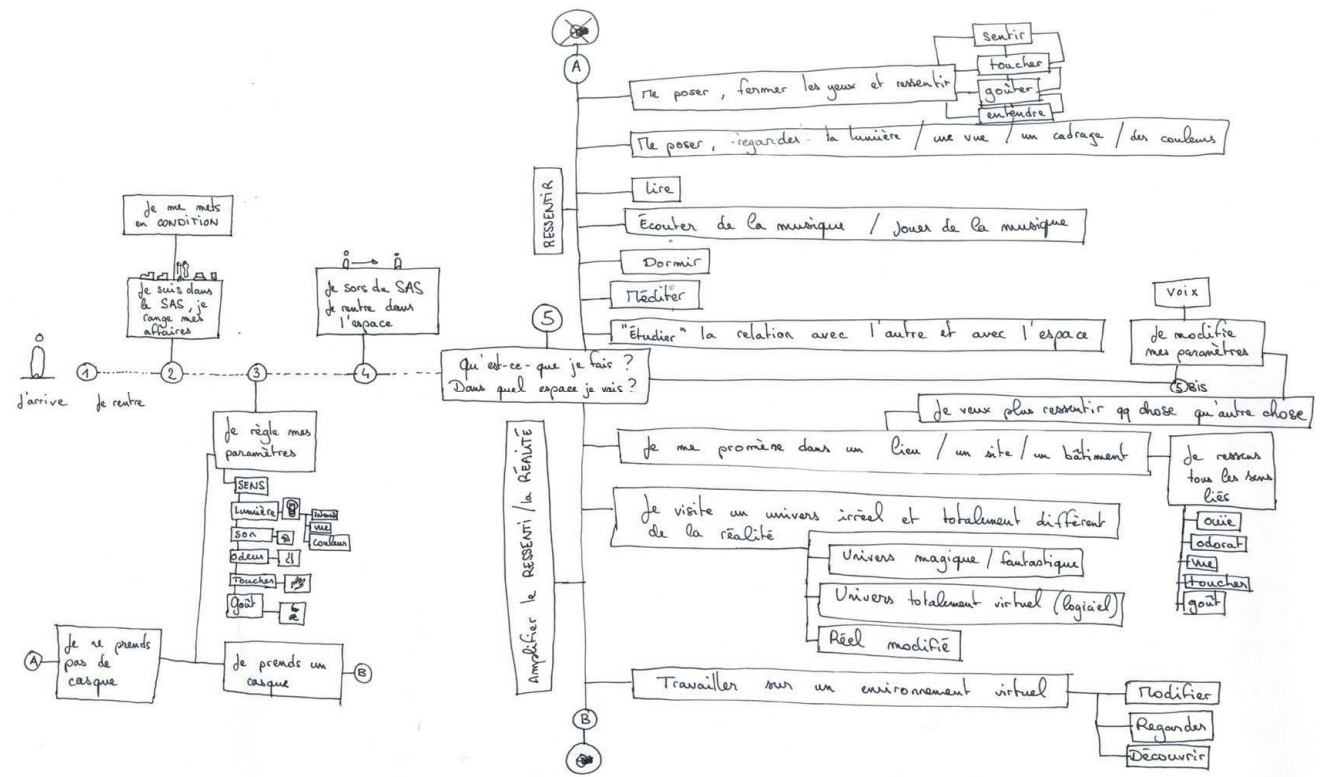

Figure 1. Diagramme des scenarii proposés pour le projet Error 404, témoignant de la pratique réflexive dans la manière d'aborder le projet

\subsubsection{EMO-Box (projet d'étudiants ${ }^{12} 2019-2020$ à l'ENSAS)}

Le projet EMO_Box - bien qu'il en soit totalement indépendant - poursuit les investigations formelles et sensorielles d'Error 404. Ce «photomaton à émotions» questionne d'une part la complémentarité entre un espace physique et sa représentation virtuelle et d'autre part l'impact des émotions et des sens sur la conception et la réception d'espaces architecturés (Figure 2). En effet, ici, l'espace physique - la box - est indissociable de l'espace virtuel dont il est la représentation. La box fait office de seuil entre ces deux dimensions. Au début de l'expérimentation, l'utilisateur prend place dans un espace particulier mais stable qu'il retrouvera à l'identique une fois équipé des lunettes de réalité virtuelle ${ }^{13}$. Cette copie conforme de l'espace physique permet d'atténuer la sensation d'ailleurs et de distanciation avec sa physicalité. Une fois immergé, l'utilisateur va faire évoluer son environnement à l'aide d'un appareil de monitoring Muse qui capte en temps réel les variations des ondes cérébrales (EEG), de l'activité cardiaque (VRC), de l'activité respiratoire, $\ldots{ }^{14}$ L'ensemble des données recueillies modifie automatiquement l'espace virtuel en faisant évoluer la luminosité, les couleurs, les formes, ...

Ainsi, l'usager projette sur l'espace ses sensations/émotions et doit procéder à un travail de méditation pour le faire évoluer, comme l'a fait Luc Schuitten avec son Arbre de lévitation $^{15}$. Avant de quitter l'environnement immersif, l'espace virtuel reprend sa forme

${ }^{11}$ Pour plus d'informations sur ce projet voir : http://www.dune-eole.fr/files/2019/11/Rendu-Master1ENSAS-14-janvier-2019-1.pdf

${ }^{12}$ LEPAUL Romain, MORNET Colas, SARGENTI Justin, SCHNINDLER Martial \& SCHWEITZER Joseph

${ }^{13}$ Voir à ce sujet l'épisode 2 de la saison 3 de Black Mirror, Playtest.

${ }^{14} \mathrm{https}: / /$ choosemuse.com/fr/muse-2/

${ }^{15}$ L'arbre de lévitation - Luc SCHUITEN - http://www.vegetalcity.net/topics/larbre-de-levitation/ 
initiale afin de permettre une transition de retour fluide. Une variante du projet est en cours, procédant de manière inverse pour comprendre comment l'espace influence l'individu.

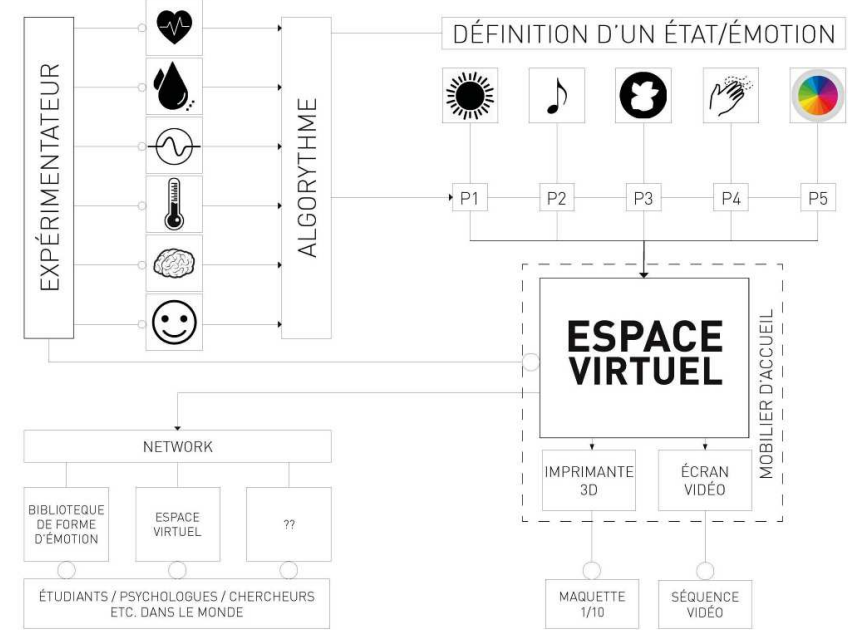

Figure 2. Diagramme conceptuel des liens entre données biologiques, définition d'un état/émotion et spatialité virtuelle dans le projet $E M O \_B o x$

\subsubsection{Premières conclusions des expérimentations étudiantes}

Les deux projets ont des similitudes dans les pratiques d'apprentissage et la réception de la réalité virtuelle par les étudiants ainsi qu'une approche très sensible dans la création d'espaces architecturés. On y retrouve la volonté de dépasser les dimensions visuelles et auditives, pour expérimenter les autres sens de différentes manières. Toutefois, l'exploration des sens tactiles, olfactifs et gustatifs a été - comme pressenti - plus complexe. Cette difficulté a donné lieu à la mise en place de «stratégies de contournement » avec un transfert subjectif de ces dimensions vers les perceptions visuelles et haptiques, c'est-à-dire, «l'ensemble sensation tactile, kinesthésie, proprioception » (Mellet d'Huart 2001, 332-333) à l'aide de dispositifs modifiant l'espace physique. Si certaines expérimentations ont déjà réussi à intégrer la dimension olfactive «par l'usage de gaz odorifères qui n'ont rien de virtuel » (Ibidem), les étudiants à l'origine d'Error 404 ont plutôt choisi de retranscrire des sensations au travers de la physicalité de l'espace, comme avait pu le faire en son temps Iannis Xenakis avec la musique.

Dans le projet EMO_Box, l'accent est mis sur la relation corps/esprit/espace(s) plutôt que sur la question de l'habiter. De nouveau, les étudiants ont mis en avant leur subjectivité et la manière dont elle s'exprime spatialement. Contrairement à Error 404, le projet avait pour ambition de s'approcher autant que possible d'une certaine forme d'objectivité grâce aux sciences cognitives et à la psychologie (notamment la psychologie des couleurs). La question du seuil entre espaces physique et virtuel a également été abordée de manière particulièrement pertinente en évitant toute rupture, renforçant ainsi le sentiment d'immersion et une proximité «des mécanismes naturels d'appréhension du monde » (Ibidem, 333). Ce faisant, les distensions spatio-temporels qu'ils expérimentent leurs paraissent d'autant plus crédibles et acceptables car la distanciation avec l'espace physique et ses normes a été, sinon totalement, largement abolie (Ibidem).

Ces deux exemples mettent en avant l'un des risques potentiels de la réalité virtuelle. Si la notion de subjectivité n'est pas dérangeante, la transcription des cinq sens ou de l'activité biologique en signaux et en formes - d'autant plus que les informations se superposent - 
peut conduire à « une surcharge informationnelle », c'est-à-dire « un flot de données qu'un sujet humain peut difficilement transformer en information et en connaissance » (Briffaut 2012, 212). Nous présupposons que cette superposition informationnelle amène certes à des espaces complexes, interactifs et généralement intéressants mais peut également faire perdre la lisibilité et la compréhension de l'espace. Comment en effet répondre à un programme spécifique ou même analyser le résultat à partir de cela?

\section{Expérimentation d'un EVE pour la co-création spatiale et pédagogique au sein d'une ENSA}

\subsection{Objectif}

Même si cela semble évoluer, nous retrouvons encore peu l'utilisation des technologies immersives dans le domaine universitaire. Nous avons donc adopté une approche expérimentale pour développer un EVE au sein des ENSA. Cette attitude empirique s'explique également par la nécessité de considérer ces environnements immersifs (et numériques) au cas par cas afin qu'ils répondent 1) à des pratiques pédagogiques spécifiques ; 2) à des attentes et à des pratiques d'apprentissage particulières des étudiants et de leurs champs disciplinaires et 3 ) à des espaces physiques et des moyens financiers mis à disposition. Sur la base des résultats des travaux étudiants, nous avons trouvé pertinent de développer un EVE - certes plus académique - mais toujours dans l'objectif de développer et d'adapter les pratiques pédagogiques pour un public majoritairement de la "génération $\mathrm{Z} »$ qui n'a connu qu'une société fondée sur un outillage et une culture numérique prédominante. L'espace développé a une triple ambition. La première consiste à poser les bases d'un outil immersif de conception des ambiances d'apprentissage qui a pour vocation d'évoluer suite aux retours des étudiants et des enseignants-chercheurs. La deuxième vise à expérimenter les interactions avec des données quantitatives et qualitatives dans un environnement virtuel en lien avec l'espace physique dans une volonté de travail sur les séquences d'apprentissage. Enfin la dernière ambition est plus générale et concerne aussi bien l'apprentissage spécifique aux études d'architecture que les études universitaires dans leur ensemble. Cet EVE est une prolongation expérimentale des réflexions menées lors de la mission de recherche Dune-EOLE sur l'impact du numérique dans l'enseignement et l'apprentissage afin de valoriser un apprentissage plus didactique, autonome, collaboratif et centré sur la notion de Learning by doing.

\subsection{Méthodologie}

Nous nous sommes basé sur la norme ISO 9241-210:2019 «Ergonomie de l'interaction homme-système - Partie 210 : Conception centrée sur l'opérateur humain pour les systèmes interactifs » qui définit cinq critères pour cadrer le développement d'un EVE : «1) La prise en compte en amont des utilisateurs, de leurs tâches et de leur environnement ; 2) La participation active des utilisateurs, garantissant la fidélité des besoins et des exigences liées à leurs tâches ; 3) La répartition appropriée des fonctions entre les utilisateurs et la technologie ; 4) L'itération des solutions de conception, jusqu'à satisfaction des besoins et des exigences exprimés par les utilisateurs et 5) L'intervention d'une équipe de conception multidisciplinaire, visant une expérience utilisateur optimale ${ }^{16}$.

\footnotetext{
${ }^{16}$ Définition Wikipédia « Conception centrée sur l'utilisateur », https://fr.wikipedia.org/wiki/Conception_centr\%C3\%A9e_sur_1\%27utilisateur
} 
Les lieux, les enseignements, les ressources et les acteurs du Laboratoire Lumière de l'ENSAS constituent le dispositif pédagogique. Équipé d'appareils de prise de mesures (luxmètres, ...), d'outils informatiques et de réalité virtuelle, de Lumbox (maquettes 1/20) et récemment d'un simulateur d'éclairage naturel (ciel artificiel et soleil mécanique) ; associant chercheurs, enseignants et étudiants, il ancre la recherche dans la pédagogie.

Dans une logique de complémentarité entre les lieux et les outils, il est d'abord demandé aux étudiants d'effectuer des relevés in-situ (dimensionnement, quantification et qualification des ambiances de l'objet d'étude). Les premières analyses se poursuivent à partir de maquettes physiques et modèles informatiques. Elles débouchent ensuite sur des intentions de projet (dispositifs architecturaux, matériaux, orientation, ...) et des hypothèses qui mènent à l'expérimentation. En plus des expérimentations des étudiants, une mission a été proposée (à la société Echoes.Paris) en vue de créer des prototypes d'interfaçage entre la réalité physique d'un lieu existant et un environnement immersif en réalité virtuelle.

\subsection{Présentation des prototypes d'interfaçage}

\subsubsection{Synchronisation semi-automatique}

La première méthodologie mise en place consiste en un protocole semi automatisé unidirectionnel entre les plateformes Grasshopper et Twinmotion/UnrealEngine. Il est d'abord demandé aux étudiants de retranscrire leurs intentions de projet en paramètres modélisables. A ce stade de développement, le protocole permet de créer des modèles paramétriques afin de générer des variantes dans les propositions de projet ou dans l'étude des ambiances (images de simulations calculées en fausses couleurs) depuis l'interface Grasshopper et de les exporter dynamiquement dans Twinmotion. L'immersion se fait ensuite grâce à un casque de réalité virtuelle.

Ce type de synchronisation ne permet pas à l'étudiant de modifier son projet lorsqu'il est immergé. Cependant, les différentes variantes générées en amont, peuvent être explorées en aval par phases. Tout en étant immergé, l'étudiant peut, d'une part, faire défiler les différentes propositions spatiales via le menu interactif ; d'autre part, superposer les informations quantitatives (images de simulation) aux informations qualitatives (l'espace du projet dans sa morphologie, avec ses matériaux, ...) (Figure 3).

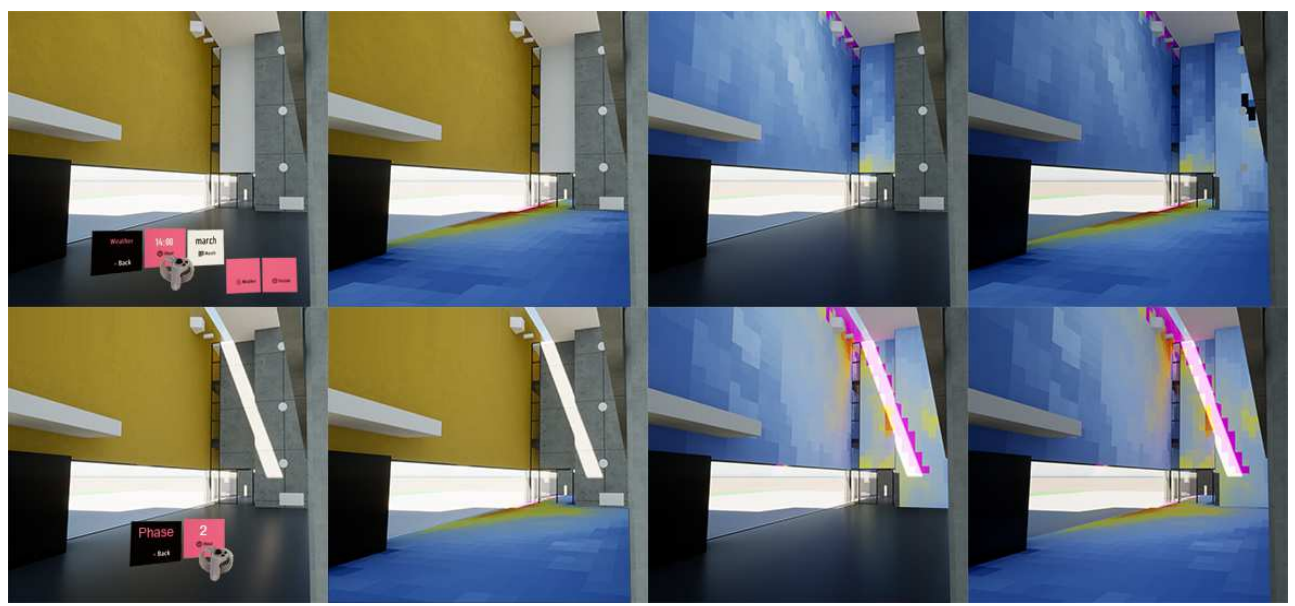

Figure 3. Variations d'ambiances en réalité virtuelle (Twinmotion synchronisé avec Grasshopper) : exploration des propositions de projet et superposition des informations qualitatives et quantitatives 


\subsubsection{Synchronisation automatique}

Comme nous l'avons vu avec le projet EMO_Box, l'une des conditions favorisant l'immersion réside dans la continuité et la fluidité entre espaces physique et virtuel. Ainsi, nous prototypons également des solutions basées sur des protocoles d'échanges d'informations en temps réel entre Grasshopper et les plateformes UnrealEngine et Unity, utilisant la technologie Rhino.Inside et des développements OpenSource.

Nous espérons pouvoir maximiser le sentiment d'immersion dans l'espace modélisé et permettre aux étudiants d'interagir avec l'environnement virtuel comme ils le feraient naturellement avec une maquette. Toutefois, la différence entre ces deux pratiques (outre les techniques et technologies utilisées) réside dans le fait que dans la manipulation de la maquette, la création de l'espace se fait du dehors lorsque l'EVE permettrait de le créer du dedans : de concevoir, fabriquer ou vérifier l'espace depuis l'espace.

Une autre particularité réside dans le fait que l'environnement permet d'interagir avec des données objectives ou subjectives. Les premières sont mesurables (géométriques ou climatiques), les secondes résultent de la sémantique du lieu d'étude, identifiées in-situ par les étudiants lorsqu'ils expriment des qualificatifs d'ambiance (lumière chaleureuse, intime, ...). L'idée est d'immerger l'étudiant dans un projet paramétrique informé, capable d'évoluer par le biais de commandes interactives (Figure 4). Si l'implémentation des données objectives est opérationnelle, la retranscription des données subjectives en données interactives est encore au stade de réflexion.

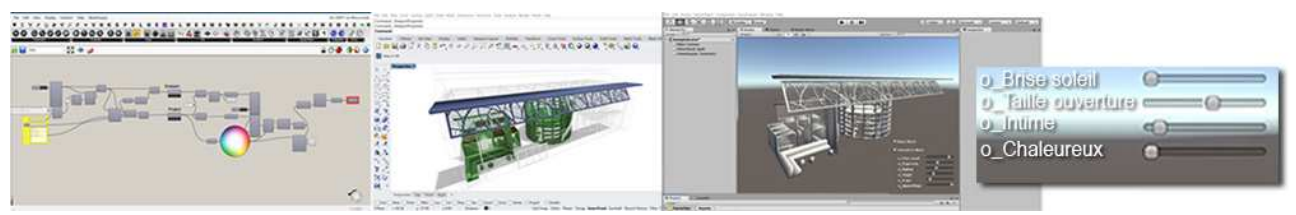

Figure 4. Synchronisation automatique Grasshopper/Rhino/Unity : interaction avec des données objectives ou subjectives en immersion virtuelle

\subsubsection{Résultats attendus}

En implémentant des liaisons dynamiques entre la plateforme Grasshopper et une plateforme de réalité virtuelle, nous souhaitons proposer un outil de programmation visuelle et de simulation d'ambiances directement manipulable en réalité virtuelle.

En superposant et manipulant des informations - qualitatives/quantitatives sensibles/physiques, nous essayons de construire un environnement sémiotique et phénoménologique, dans lequel l'étudiant/apprenant (en tant que designer comme autoorganisateur) peut appréhender les ambiances qui en résultent dans une perception dynamique et rythmée par ses actions ; ce qui aurait pour conséquence de réduire le risque de « surcharge informationnelle ».

L'expérience vécue ne fait sens que dans le cadre du dispositif pédagogique, avec la mise en commun et l'interprétation des résultats dans une logique réitérative faisant intervenir l'ensemble des participants, c'est-à-dire, étudiants, enseignants et chercheurs.

Les premiers prototypes seront testés au deuxième semestre ${ }^{17}$ (février-juin 2020) dans l'atelier optionnel de Master 1 «Laboratoire Lumière: ambiances et immersions » à l'ENSAS, afin d'avoir des retours sur la réception et l'usage qu'en font les étudiants et les enseignants-chercheurs. Cette phase de tests permettra d'améliorer l'outil afin de pouvoir, à

${ }^{17}$ Dans le cadre de la crise sanitaire liée au Coronavirus, les expérimentations sont limitées 
terme, le pérenniser et permettre à tous les étudiants d'y avoir accès. Ce projet pilote pourrait également servir de base à d'autres cursus universitaires.

\section{Conclusion}

«Une des choses que nos petits-enfants trouveront le plus bizarre à notre sujet, c'est que nous faisons la différence entre le numérique réel, entre le virtuel et le réel. Dans le futur, cela serait littéralement impossible. La distinction entre le cyberespace et ce qui n'est pas le cyberespace va devenir inimaginable. » (Gibson, 2007)

Cette phrase de William Gibson, l'auteur de l'ouvrage culte Neuromancien résume l'un des premiers constats de ces expérimentations. Après une première phase de compréhension de l'environnement immersif, de ces potentialités, de ces limites et de premiers tâtonnements, les étudiants ont vite intégrés d'eux-mêmes qu'aucun des espaces physique et virtuel n'étaient substituables ou antagonistes mais bien complémentaires dans les modalités de conception et d'usage. Cela a confirmé l'intérêt et les intentions des enseignants-chercheurs de travailler par séquences d'apprentissage: la conception commence dans l'espace physique et se poursuit dans l'espace virtuel. Il est également apparu un besoin commun de lier l'expérience virtuelle à une expérience sensible, sensorielle, dans les différentes expérimentations. Aussi, nous nous sommes rendus compte que les étudiants ont considéré la réalité virtuelle comme un espace d'apprentissage et d'expérimentations et non pas comme un outil de visualisation ou de rendus.

La mise en œuvre d'un EVE au sein de l'ENSAS - bien qu'encore au stade expérimental - a déjà amené des résultats très encourageants et soulevé de nombreuses problématiques. Plus qu'un simple outil de «vérification » ou de rendu, l'utilisation libre de l'EVE par les étudiants a mis en exergue des questions fondamentales dans la conception d'espaces d'apprentissage et dans l'apprentissage de l'architecture : spatialité et temporalité de l'architecture ; liens entre espaces physique et virtuel ; rapport entre corps, émotions et espaces; influence de l'outil sur le processus de conception ; réflexivité, autodidaxie et adaptation des pratiques; séquences d'apprentissage; place des sens dans l'élaboration du projet, ...

Les résultats des expérimentations montrent également la manière dont l'usage d'environnements immersifs et le séquençage de l'apprentissage entre les espaces physique et virtuel amènent à (re)questionner les modalités d'interaction, d'enseignement et d'apprentissage (Loup-Escande, Jamet et al. 2015, 1). Il apparaît en effet qu' « une réflexion sur la formation doit notamment appréhender la complexité du processus cognitif de transfert de connaissances lequel nécessite une décontextualisation puis une recontextualisation (Fallery \& Marti, 2016) et met le rôle de l'enseignant au premier plan » (Weber, Rodhain \& Fallery 2019, 39). De plus, comme l'ont mis en évidence David Gauckler et Jean-François Cerisier, s'il est vrai que les étudiants de la « génération Z nés avec le numérique, cela ne présuppose en rien leur capacité «à manipuler les objets techniques numériques [qui] induirait une évidence à l'usage des environnements immersifs » (Gauckler 2019, 9). Il convient plutôt de considérer que cette génération (comme la société en général) a été impactée - transformée - par le numérique d'abord et essentiellement d'un point de vue culturel et social plus que d'un point de vue technique. Toutefois, un regard critique sur la culture numérique ne va pas de soi et n'ai que très peu abordé dans l'enseignement numérique alors même qu'il semble incontournable.

\section{Bibliographie}


Albero, B. (2010a). Une approche sociotechnique des environnements de formation. Rationalités, modèles et principes d'action, in Education \& Didactique [En ligne], 2010, Vol 4, n 1, 7-24. https://doi.org/10.4000/educationdidactique.715

Albero, B. (2010b). La formation en tant que dispositif : du terme au concept. B. Charlier et F. Henri(dir. par). La technologie de l'éducation : recherches, pratiques et perspectives, Presses Universitaires de France (pp.47-59). [En ligne] https://edutice.archivesouvertes.fr/edutice-00578663

Briffaut, J. (2012). Apprentissage, expertise et réalité virtuelle du point de vue de la prise de décision avec l'éclairage des neurosciences. Prospective et stratégie, "L'expertise ", numéros 2-3(1), 197-217, APORS Éditions. doi:10.3917/pstrat.002.0197.

Descamps, N. (2017). De l'algorithme à la ville, vers un Minecraft IRL, mémoire de M2, ENSAS, https://issuu.com/nicolasdescamps9/docs/del_algorithme_lavillenicolasdesca

Descamps, N. (2018). Etat de l'art des Nouveaux Espaces d'Apprentissage (NEA), Mission de Recherche Projet DUNE-EOLE, ANR-16-DUNE-0001-EOLE, ENSA Strasbourg. http://www.dune-eole.fr/actualites/\#page-content

Delépine, O. (2018). L'atelier en tant qu'espace et mode d'enseignement du projet en école d'architecture. Interrogations sur l'introduction du numérique. L'expérience de l'Ecole Nationale d'Architecture de Bretagne (ENSAB Rennes), in Modèles de formation et architecture dans l'enseignement supérieur. Albero B., Yurèn, T., Guérin J. (dir), Dijon : éditions Raison et Passions, Chapitre 11 (pp. 241-254).

Fallery, B., \& Marti C. (2016). «Le storytelling : un outil de gestion des connaissances », Systèmes d'Information et Management, 12(4).

Gauckler, D. (2019). Approche diachronique et réflexions stratégiques sur les espaces virtuels immersifs dans un cadre pédagogique, Etude sur les espaces virtuels immersifs, Projet DUNE-EOLE. Université de Strasbourg.

Gibson, W. (2007). William Gibson: The Rolling Stone interview, Rolling Stone ${ }^{\circ} 1039,15$ novembre 2007 (p.162).

Jones, J.C. (1970). Design Methods. Hoboken : Editions John Wiley \& Sons.

Loup-Escande, E., Jamet, E., Ragot, M., Erhel, S., Michinov, N., Peltier, C., Lopez, Th. (2015). Concevoir des environnements virtuels éducatifs avec les utilisateurs finaux : Exemple du projet VirtualiTeach. Terminal [En ligne] $\mathrm{n}^{\circ} 117$, (pp.1-19). http://journals.openedition.org/terminal/1093.

Mazalto, M., Paltrinieri, L. (2013). Introduction: Espaces scolaires et projets éducatifs, Revue internationale d'éducation de Sèvres, 64 (pp.31-40).

Mellet d'Huart, D. (2001). La réalité virtuelle : un média pour apprendre. Cinquième colloque Hypermédias et apprentissages (pp.331-338). Grenoble, France.

Merchant Z., Goetz E.T., Cifuentes L., Keeney-Kennicutt W. \& Davis T.J. (2014). "Effectiveness of virtual reality-based instruction on students' learning outcomes in K12 and higher education : A meta-analysis", Computers \& Education, 70, 29-40, Elsevier.

Rabardel, P. (1995). Les hommes et les technologies, approche cognitive des instruments contemporains, Paris, Armand Colin.

Touvenot, E., Varano, S. (2019). Les espaces d'apprentissage, action 1.1. Faire évoluer les espaces physiques. Projet ANR-16-DUNE-0001-EOLE. http://www.duneeole.fr/actualites/\#page-content

Valenzuela-Aguilera, A. (2018). Habiter l'espace hybride, in Modèles de formation et architecture dans l'enseignement supérieur. Albero B., Yurèn, T., Guérin J. (dir), Dijon : éditions Raison et Passions, Préface (pp. 11-15).

Weber, M., Rodhain, F. \& Fallery, B. (2019). Usage de la réalité virtuelle et développement individuel des enseignants-chercheurs. Une approche par la didactique professionnelle. Management \& Avenir, 112(6), 37-57. doi:10.3917/mav.112.0037. 